\title{
Erratum to: Linear Time Algorithms for Generalized Edge Dominating Set Problems
}

\author{
André Berger • Ojas Parekh
}

Received: 25 May 2011 / Accepted: 27 July 2011 / Published online: 25 August 2011

(C) Springer Science+Business Media, LLC 2011

\section{Erratum to: Algorithmica (2008) 50(2):244-254 \\ DOI 10.1007/s00453-007-9057-y}

In our paper "Linear Time Algorithms for Generalized Edge Dominating Set Problems" [1] we claim that the edge-edge adjacency matrix with one's on the diagonal is totally unimodular (Lemma 6). Unfortunately there is a mistake in the proof of Lemma 6 and Fig. 1 shows a counterexample to the lemma.

The matrix in question is

$$
M=\left(\begin{array}{llllll}
1 & 1 & 1 & 0 & 1 & 0 \\
1 & 1 & 0 & 0 & 0 & 0 \\
1 & 0 & 1 & 1 & 1 & 0 \\
0 & 0 & 1 & 1 & 0 & 0 \\
1 & 0 & 1 & 0 & 1 & 1 \\
0 & 0 & 0 & 0 & 1 & 1
\end{array}\right)
$$

and its determinant is 2 . This shows that the edge-edge adjacency matrix with one's on the diagonal is in general not totally unimodular for all trees.

The online version of the original article can be found under doi:10.1007/s00453-007-9057-y.

We would like to thank Keiko Imai, Naoyuki Kamiyama and Yusuke Matsumoto for pointing out the mistakes in the published version and for providing the counterexample.

\footnotetext{
A. Berger $(\bowtie)$

Department of Quantitative Economics, Maastricht University, P.O. Box 616, 6200 MD Maastricht, The Netherlands e-mail: a.berger@maastrichtuniversity.nl

O. Parekh

Sandia National Laboratories, MS 1316, Albuquerque, NM 87185, USA

e-mail: odparek@sandia.gov
} 
Fig. 1 A counterexample to

Lemma 6

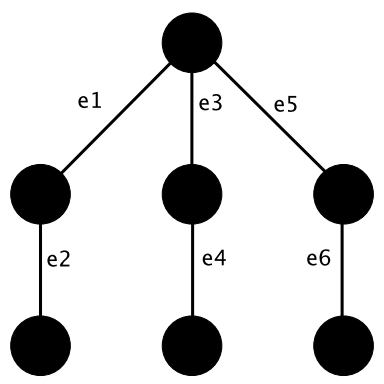

Theorem 1, based on Lemma 6, then concludes that the $b$-EDS problem on weighted trees can be solved optimally in strongly polynomial time for any values of $b$. At this time we do not have a different proof for or a disproof to Theorem 1 .

Moreover, we overlooked a technical detail in the description of Algorithm 1. In Line 6 of the algorithm, for certain edges which are incident upon the vertex $w$, a child edge of that edge is added to the set $S$. However, there may exist a child $s$ of $w$ which is a leaf itself and for which $b(w s)=1$. If such an edge $w s$ exists then one just has to compute $F=E D S(T-g \operatorname{ch}(w), b, c)$ instead of the steps in Lines 6-13. Using this modification of the algorithm renders Theorem 3 correct.

\section{References}

1. Berger, A., Parekh, O.: Linear time algorithms for generalized edge dominating set problems. Algorithmica 50(2), 244-254 (2008) 